\title{
Impact of Preharvest Sprouting of Wheat (Triticum aestivum) in the Field on Starch, Protein, and Arabinoxylan Properties
}

\author{
Heleen Olaerts, ${ }^{\dagger}$ Chiara Roye, ${ }^{\dagger}$ Liesbeth J. Derde, ${ }^{\dagger}$ Georges Sinnaeve, ${ }^{\ddagger}$ Walter R. Meza, ${ }^{\S}$ \\ Bernard Bodson, ${ }^{\S}$ and Christophe M. Courtin* ${ }^{\dagger}$

\begin{abstract}
${ }^{\dagger}$ Laboratory of Food Chemistry and Biochemistry, and Leuven Food Science and Nutrition Research Centre (LFoRCe), KU Leuven, Kasteelpark Arenberg 20, B-3001 Leuven, Belgium

*Département Valorisation des Productions, Centre Wallon de Recherches Agronomiques (CRA-W), Chaussée de Namur 24, B-5030 Gembloux, Belgium
\end{abstract} \\ ${ }^{\S}$ Unité de Phytotechnie des Régions Tempérées, Gembloux Agro-Bio Tech (G-ABT), Université de Liége, Passage des Déportés 2, \\ B-5030 Gembloux, Belgium
}

ABSTRACT: To obtain detailed knowledge on possible changes in the properties of starch, proteins, and arabinoxylan as a result of field preharvest sprouting (PHS), three wheat varieties were harvested at maturity and several weeks later when severe PHS had occurred. Falling number values of flour dropped from 306 to $147 \mathrm{~s}$ (Sahara), 382 to $155 \mathrm{~s}$ (Forum), and 371 to $230 \mathrm{~s}$ (Tobak). Blocking of $\alpha$-amylase activity demonstrated that the decline in falling number and changes in RVA pasting and gelation properties were not caused by changes in intrinsic starch properties as a result of PHS. PHS had no influence on the SDS-extractability and molecular weight distribution of the proteins. For arabinoxylan, incipient breakdown was noticed, leading to a higher amount and average degree of polymerization of water extractable arabinoxylan. Results show that strategies to cope with severely PHS in wheat should focus on blocking enzyme activities.

KEYWORDS: field sprouting, cereal constituents, germination, hydrolytic enzymes

\section{INTRODUCTION}

Wheat is an important raw material for production of staple food in the Western world. Therefore, variations in yield and quality are of major concern for farmers and the wheat processing industry. Such variations are often caused by varying climatological conditions shortly before harvest. In 2014, heavy rainfalls resulted in numerous wheat crops all over Europe being adversely affected by sprout damage, leading to considerable variation in wheat quality. Preharvest sprouting (PHS) of wheat is the germination of wheat kernels in the ear of the parent plant in the field and follows upon periods of prolonged and repeated rainfalls on grain shortly before or during harvest. This phenomenon is well-known in the wheat industry as it results in a decreased yield and an impaired quality of the grain. ${ }^{1}$ For example, flour from preharvest sprouted wheat produces dough which is sticky, insufficiently elastic, and difficult to handle, hence creating bread with a sticky and gummy texture, low loaf volume, and a darker crust color. $^{2-5}$ As end-products made of preharvest sprouted wheat are generally unacceptable to consumers, sprouted grains are often downgraded to feed grain.

PHS is accompanied by various physiological changes occurring in the grain which are of major importance for a food processing point of view. It has been commonly assumed that laboratory germinated grain can be used as a model to study field sprout damage, nevertheless the conditions of germination differ. In those studies, deteriorated end product quality due to PHS damage is, on the one hand, caused by the increased activity of hydrolytic enzymes formed during germination and, on the other hand, due to altered inherent properties of starch, gluten, and arabinoxylan (AX) within the grain. ${ }^{1,6} \alpha$-Amylases formed during germination cause erosion of starch granules starting at the endosperm-embryo junction in the kernel, resulting in increased content of damaged starch and soluble sugars. ${ }^{5,7-9}$ This degradation subsequently alters physicochemical properties noticeable by a decrease in water binding capacity and swelling power, and a decreased gelatinization temperature of starch over a more narrow temperature range. ${ }^{9,10}$ In addition, due to hydrolysis of peptide bonds a decrease in molecular weight of proteins and a rapid increase in free amino groups was observed, resulting in modified solubility properties of the proteins. ${ }^{2,11,12}$ Already during initial stages of germination, degradation of AX was detected by an increase in the amount of water extractable AX (WEAX). ${ }^{13}$ However, the above-mentioned studies have been conducted on sound wheat kernels germinated under laboratory conditions, which differ strongly from the conditions for PHS in the field.

Indeed, already in 1973, Meredith and Jenkins ${ }^{14}$ declared that laboratory sprouted grain should not be used as a proxy for field sprouting. In this context, Kulp et al. ${ }^{15}$ were one of the first to study wheat sprouted in the field and observed no changes in physicochemical properties of starch in contrast to the above studies. In addition, Olaerts et al. ${ }^{16}$ revealed that unfavorable weather conditions around harvest time largely influence

Received: July 13, 2016

Revised: October 12, 2016

Accepted: October 13, 2016

Published: October 13, 2016 
hydrolytic enzyme activities in the kernel, in a manner distinct from what was previously observed for mature kernels germination under laboratory conditions. Disparity in the results found between sprouting under laboratory conditions and in the field is not surprising as the conditions of temperature and humidity under which sprouting occurs in both situations differ largely, which cause an inefficient onset of germination in the field. In addition, this leads to a nonuniform distribution and extent of germinated kernels in the ear of the plant in the field (unpublished results), in contrast to a simultaneous germination of almost all kernels when germinating under controlled laboratory conditions. Hence, the resulting functional changes can also differ in these two kinds of germination. Consequently, more care should be taken when drawing conclusions from results conducted by laboratory sprouted wheat grain to indicate the effect of field PHS on wheat and end product quality.

To have a better understanding of the factors responsible for quality deterioration of wheat and end products made from preharvest sprouted wheat, in a previous study ${ }^{16}$ wheat samples from three different wheat varieties, with a different susceptibility for PHS, were harvested regularly from prematurity until the moment that wheat was severely sprouted in the field. These samples were used to examine the evolution of hydrolytic enzymatic activity in wheat (flour) during field PHS. In the current study, the aim is to identify possible changes that have occurred in vivo in wheat constituents as a result of PHS. To this end, a mature (as control) and a severely sprouted sample from each of the three varieties were selected, with the latter sample sprouted to such a level, i.e., a Hagberg falling number (FN) of flour of ca. $150 \mathrm{~s}$, that the wheat will typically be rejected in the food industry at time of sale. These samples were used to investigate whether structural or functional changes in starch, proteins, and AX have occurred as a result of PHS in the field. Knowledge of the properties of these substrates in field sprouted wheat will contribute to the development of postharvest treatments to upgrade severely sprouted wheat.

\section{MATERIALS AND METHODS}

Sample Collection. Three winter wheat (Triticum aestivum) cultivars (Forum, Tobak, and Sahara), with a difference in sprouting tolerance, were cultivated and harvested in 2014 as described in Olaerts et al. ${ }^{16}$ Delaying harvest time in the presence of rainy weather conditions caused PHS in the grain which was visible in a small amount of kernels by the penetration of the pericarp by the coleoptile and roots. ${ }^{16}$ In the present study, for each variety, samples with the maximal (mature wheat) and a low (severely sprouted wheat) FN in the sample range were selected. The selected mature samples were Sahara harvested at July 28 (further referred as Sahara 28/7; whole meal FN = $230 \mathrm{~s}$ ), Forum 28/7 (whole meal FN = $320 \mathrm{~s}$ ), and Tobak $1 / 8$ (whole meal FN = $330 \mathrm{~s}$ ). These mature samples were used as reference for the corresponding sprouted wheat samples: Sahara 20/8 (whole meal FN $=80 \mathrm{~s}$ ), Forum $25 / 8$ (whole meal FN = $110 \mathrm{~s}$ ), and Tobak 2/9 (whole meal FN = $190 \mathrm{~s}$ ), in which 10.5, 6.0, and $1.5 \%$, respectively, of the kernels was visibly sprouted. The six wheat samples were conditioned to $16.0 \%$ moisture and milled into flour with a Bühler MLU-202 laboratory mill (Bühler AG, Uzwil, Switzerland). Flour yield was 73 and $71 \%, 75$ and $75 \%$, and 71 and $73 \%$, for Sahara 28/7 and 20/8, Forum 28/7 and 25/8, and Tobak $1 / 8$ and 2/9, respectively. Flour samples had an ash content of 0.57 and $0.52 \%, 0.61$ and $0.52 \%$, and 0.54 and $0.49 \%$, for Sahara $28 / 7$ and 20/8, Forum 28/ 7 and $25 / 8$, and Tobak $1 / 8$ and $2 / 9$, respectively.

(Bio)chemical Analyses. Moisture content was determined according to AACC International method $44-15.02 .{ }^{17}$ Protein levels
$(\mathrm{N} \times 5.7)$ were determined using an adaptation of the AOAC official method $990-03^{18}$ to an automated Dumas protein analysis system (EAS VarioMax N/CN, Elt, Gouda, The Netherlands). Flour damaged starch levels were determined according to AACC International method $76-31-01^{17}$ using a colorimetric assay provided by Megazyme (Bray, Ireland). Total starch (measured as polymeric noncellulosic glucose) and total AX contents of the flour were determined through gas chromatographic analysis of monosaccharide content and composition after acid hydrolysis, reduction and acetylation as described by Dornez et al. ${ }^{19}$ The total amount of WEAX was determined by a similar procedure preceded by inactivation of the enzymes present with ethanol. To this end, flour $(1.0 \mathrm{~g})$ was suspended in $20.0 \mathrm{~mL}$ of $80 \%(\mathrm{v} / \mathrm{v})$ aqueous ethanol and vigorously shaken. Next, all added ethanol was evaporated at $95{ }^{\circ} \mathrm{C}$ to inactivate enzymes. Flour extracts were prepared by dissolving the dry pellet in $10.0 \mathrm{~mL} \mathrm{H}_{2} \mathrm{O}$. After shaking $\left(30 \mathrm{~min}, 7^{\circ} \mathrm{C}\right.$ ), the suspensions were centrifuged $\left(10 \mathrm{~min}, 4000 \mathrm{~g}, 7^{\circ} \mathrm{C}\right)$ and the supernatants were analyzed immediately. Reducing end xylose contents in extracts were determined by gas chromatography after reduction of the extracts followed by hydrolysis and acetylation of the resulting reduced monosaccharides. To determine free xylose, arabinose, and glucose content, the same procedure was performed, but the hydrolysis step was omitted. Total AX, WEAX, and starch levels were calculated as 0.88 times the sum of arabinose and xylose content, and 0.90 times the glucose content, respectively. When calculating the AX content, the total arabinose content was corrected for arabinose originating from arabinogalactan-peptides and free arabinose, while the xylose content was corrected for free xylose content present in the extracts. The average degree of polymerization (avDP) of WEAX was calculated as the sum of the arabinose and xylose contents (after correction of the arabinose content for the presence of arabinogalactan-peptides and free xylose and arabinose), divided by the reducing end xylose content (corrected for free xylose content). $\alpha$-Amylase and endoxylanase activities were determined and expressed in AU/g dry matter $(\mathrm{dm})$ flour or EU/g dm flour, respectively, as described before. ${ }^{16}$ All analyses were performed in triplicate and values were expressed as a proportion of the total dry weight of the analyzed sample.

Content of Mono-, Di-, and Oligosaccharides. After addition of $500 \mathrm{~mL}$ of an internal standard solution $(8.0$ rhamnose $\mathrm{mg} / \mathrm{mL}$ ), flour samples $(500.0 \mathrm{mg}$, in triplicate) were suspended in $20.0 \mathrm{~mL}$ of an $80 \%(\mathrm{v} / \mathrm{v})$ aqueous ethanol solution and shaken at $80{ }^{\circ} \mathrm{C}$ for 30 $\min$ to extract sugars and denature proteins and, hence, avoid enzymatic degradation. Suspensions were heated at $95{ }^{\circ} \mathrm{C}$ until all added ethanol was evaporated and the resulting pellet was dissolved in water $(25.0 \mathrm{~mL})$, centrifuged $(10 \mathrm{~min}, 4000 \mathrm{~g})$, and filtered. Supernatants were appropriately diluted in water and filtered $(0.22$ $\mu \mathrm{m})$. Saccharides in the different samples were analyzed with highperformance anion-exchange chromatography with pulsed amperometric detection on a Dionex ICS 3000 chromatography system (Sunnyvale, CA, USA). Samples $(25 \mu \mathrm{L})$ were injected on a CarboPac PA-100 guard column $(50 \times 4 \mathrm{~mm})$ coupled to a CarboPac PA-100 anion exchange column $(250 \mathrm{~mm} \times 4 \mathrm{~mm})$. Elution $(1.0 \mathrm{~mL} / \mathrm{min})$ was with a linear gradient of $0-400 \mathrm{mM}$ sodium acetate in $100 \mathrm{mM}$ sodium hydroxide. Calibration samples containing the internal standard and malto-oligosaccharides (glucose, maltose, maltotriose, maltotetraose, and maltopentaose) were used to identify and quantify the saccharides present in flour. All carbohydrate concentrations are expressed on a dm flour basis.

Hagberg Test. The FN was measured in triplicate according to AACC International method $56-81.03^{17}$ in a FN 1500 System (Perten Instruments, Hägersten, Zweden) and with a sample size of $7.00 \mathrm{~g}$ (14\% moisture basis) in $25 \mathrm{~mL}$ deionized water. To inactivate $\alpha$ amylases, water was replaced with the same volume of a $2.0 \mathrm{mM}$ silver nitrate solution. The FN is defined as the total time in seconds required to stir $(60 \mathrm{~s})$ and to allow a viscometer stirrer to fall a specified distance through the heated flour-water suspension.

Pasting and Gelation Properties. Flour rheological properties were studied using a Rapid Visco Analyzer (RVA) (RVA Super 4, Perten instruments). Flour samples $(12.0 \% \mathrm{dm})$ were suspended in deionized water or in silver nitrate solution $(1.0,2.0$, or $10.0 \mathrm{mM})$ 
Table 1. Hagberg Falling Number (s) Measured in Deionized Water or $2 \mathrm{mM}$ Silver Nitrate of Flour of Mature and Field Sprouted Wheat of Sahara, Forum, and Tobak ${ }^{a}$

\begin{tabular}{|c|c|c|c|c|c|c|}
\hline & Sahara $28 / 7$ & Sahara $20 / 8$ & Forum $28 / 7$ & Forum $25 / 8$ & Tobak $1 / 8$ & Tobak 2/9 \\
\hline falling number with deionized water $(\mathrm{s})$ & $306 \pm 6 \mathrm{~A}$ & $147 \pm 1 \mathrm{~B}$ & $382 \pm 4 \mathrm{~A}$ & $155 \pm 3 \mathrm{~B}$ & $371 \pm 2 \mathrm{~A}$ & $230 \pm 5 B$ \\
\hline falling number with $2 \mathrm{mM}$ silver nitrate (s) & $438 \pm 6 \mathrm{~A}$ & $443 \pm 6 \mathrm{~A}$ & $458 \pm 11 \mathrm{~A}$ & $451 \pm 7 \mathrm{~A}$ & $414 \pm 5 \mathrm{~A}$ & $424 \pm 6 \mathrm{~A}$ \\
\hline
\end{tabular}

${ }^{a}$ Values are averages with standard deviations on triplicate measurements. Values of same variety in the same row of the table followed by a different letter are significantly different $(p<0.05)$.
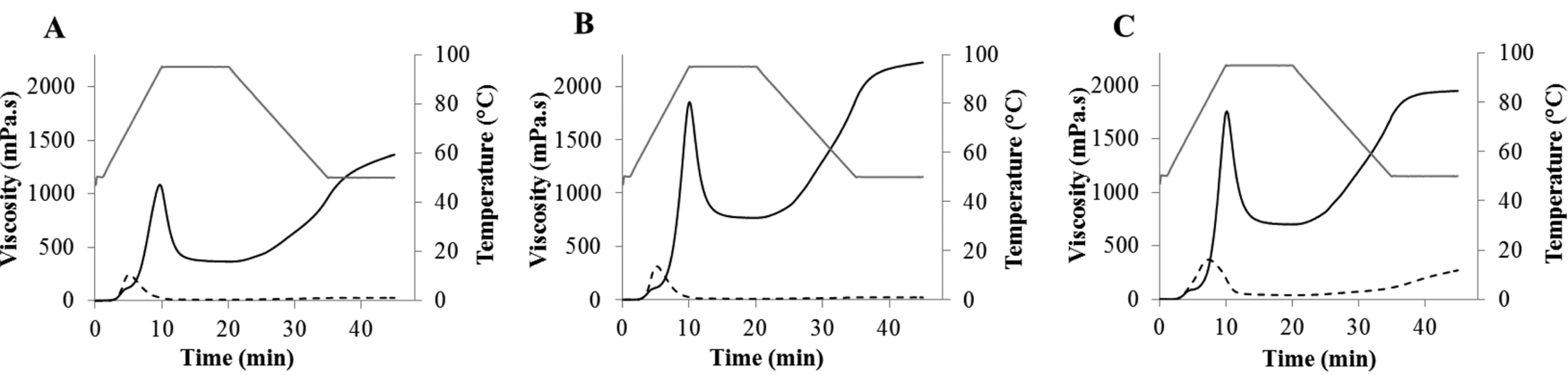

Figure 1. RVA pasting profiles of flour $(12 \% \mathrm{dm})$ of mature (black line) and field sprouted (dotted line) wheat of Sahara (A), Forum (B), and Tobak (C). The applied temperature profile is shown with a gray line.

Table 2. RVA Peak Temperature and Peak, Minimum and End Viscosity Readings of Flour of Mature and Field Sprouted Wheat of Sahara, Forum, and $\operatorname{Tobak}^{a}$

\begin{tabular}{|c|c|c|c|c|c|c|}
\hline & Sahara $28 / 7$ & Sahara $20 / 8$ & Forum $28 / 7$ & Forum $25 / 8$ & Tobak $1 / 8$ & Tobak $2 / 9$ \\
\hline peak temperature $\left({ }^{\circ} \mathrm{C}\right)$ & $94 \pm 1 \mathrm{~A}$ & $70 \pm 1 \mathrm{~B}$ & $95 \pm 1 \mathrm{~A}$ & $71 \pm 1 \mathrm{~B}$ & $95 \pm 1 \mathrm{~A}$ & $82 \pm 1 \mathrm{~B}$ \\
\hline peak viscosity (mPa.s) & $1084 \pm 13 \mathrm{~A}$ & $244 \pm 4 B$ & $1854 \pm 21 \mathrm{~A}$ & $311 \pm 6 \mathrm{~B}$ & $1756 \pm 17 \mathrm{~A}$ & $371 \pm 4 \mathrm{~B}$ \\
\hline minimum viscosity (mPa.s) & $365 \pm 10 \mathrm{~A}$ & $8 \pm 2 B$ & $768 \pm 14 \mathrm{~A}$ & $9 \pm 3 \mathrm{~B}$ & $699 \pm 4 \mathrm{~A}$ & $38 \pm 3 \mathrm{~B}$ \\
\hline end viscosity (mPa.s) & $1363 \pm 19 \mathrm{~A}$ & $26 \pm 3 \mathrm{~B}$ & $2224 \pm 16 \mathrm{~A}$ & $24 \pm 5 B$ & $1947 \pm 5 \mathrm{~A}$ & $271 \pm 12 \mathrm{~B}$ \\
\hline
\end{tabular}

${ }^{a}$ Values are averages with standard deviations on triplicate measurements. Values of same variety in the same row of the table followed by a different letter are significantly different $(p<0.05)$.

until a total mass of $25.0 \mathrm{~g}$. Next, the suspensions were equilibrated for $1 \mathrm{~min}$ at $50{ }^{\circ} \mathrm{C}$, heated to $95{ }^{\circ} \mathrm{C}$ at $5{ }^{\circ} \mathrm{C} / \mathrm{min}$, followed by an isothermal holding step at $95^{\circ} \mathrm{C}$ for $10 \mathrm{~min}$, cooled to $50{ }^{\circ} \mathrm{C}$ at $3{ }^{\circ} \mathrm{C} /$ $\mathrm{min}$, and finally held at $50{ }^{\circ} \mathrm{C}$ for $10 \mathrm{~min}$. The stirring speed was 160 $\mathrm{rpm}$ and viscosity was expressed in $\mathrm{mPa}$.s. The pasting temperature is that at the onset of the viscosity rise. All analyses were performed in triplicate.

Protein Extractability in Sodium Dodecyl Sulfate Containing Medium. Changes in molecular weight distribution and extractability in a sodium dodecyl sulfate (SDS) containing medium were measured with size exclusion high-performance liquid chromatography (SEHPLC). Wheat flour was extracted $(60 \mathrm{~min})$ in triplicate with a $0.05 \mathrm{M}$ sodium phosphate buffer ( $\mathrm{pH} 6.8$ ) containing $2.0 \%(\mathrm{w} / \mathrm{v}$ ) SDS (Acros Organics, Geel, Belgium). The quantity of buffer used was $1.0 \mathrm{~mL}$ per $1.0 \mathrm{mg}$ protein in the sample. To determine protein extractability under reducing conditions, samples were extracted under nitrogen atmosphere with the SDS buffer containing $2.0 \mathrm{M}$ urea and 1.0\% (w/ v) dithiothreitol (Acros Organics, Geel, Belgium). Suspensions were centrifuged $(10 \mathrm{~min}, 10,000 \mathrm{~g})$ and filtered $(0.45 \mu \mathrm{m}$, Millex-GP, polyethersulfone, Millipore). The protein extracts were subsequently separated with SE-HPLC using an LC-2010 HT system (Shimadzu, Kyoto, Japan) with automatic injection. The extracts $(20 \mu \mathrm{L})$ were loaded on a Yarra-SEC-S4000 column (Phenomenex, Torrance, CA, USA). The elution solvent was sodium phosphate buffer (0.05 M, pH 6.8) with a flow rate of $1.0 \mathrm{~mL} / \mathrm{min}$ and the column temperature was $30^{\circ} \mathrm{C}$. Eluted protein was detected at $214 \mathrm{~nm}$. The amount of proteins extractable in SDS containing buffer, further referred to as SDSEP, was calculated from the area under the SE-HPLC chromatogram and expressed as a percentage of the total amount of protein, i.e., the amount of extractable proteins from the corresponding sample under reducing conditions.
Statistical Analyses. Significant differences for several variables, based on at least three individual measurements, were determined by one-way analysis of variance using JMP Pro software 11.0.0 (SAS Institute, Cary, NC, USA), with comparison of mean values using the Tukey test $(\alpha=0.05)$.

\section{RESULTS}

FN of Flour from Sound and Sprouted Wheat. FN values for flour from mature kernels of Sahara, Forum, and Tobak were 306, 382, and $371 \mathrm{~s}$, respectively (Table 1). Sahara flour was characterized by a lower FN value due to the presence of higher $\alpha$-amylase activity levels. ${ }^{16}$ Delaying harvest time caused a significant reduction in FN to 147,155 , and $230 \mathrm{~s}$ for flour of sprouted wheat of Sahara, Forum and Tobak, respectively. Addition of $2 \mathrm{mM}$ silver nitrate to inhibit $\alpha$ amylases led to a major rise in FN values in both sound and sprouted wheat samples, which were not significantly different from each other anymore (Table 1).

Pasting and Gelation Behavior of Flour from Sound and Sprouted Wheat. To study differences in pasting and gelation behavior of flour from sound and sprouted wheat, flour suspensions were submitted to a time-temperature profile in the RVA (Figure 1 and Table 2). Flour of wheat at harvest maturity was associated with high peak, minimum and end viscosity values, which were, like FN values, slightly lower for Sahara due to the higher $\alpha$-amylase activity. ${ }^{16}$ The temperature at which maximum viscosity was obtained during the test, was around $94-95{ }^{\circ} \mathrm{C}$ and similar for the three varieties. This peak temperature markedly shifted to lower values, 70,71 , and 82 

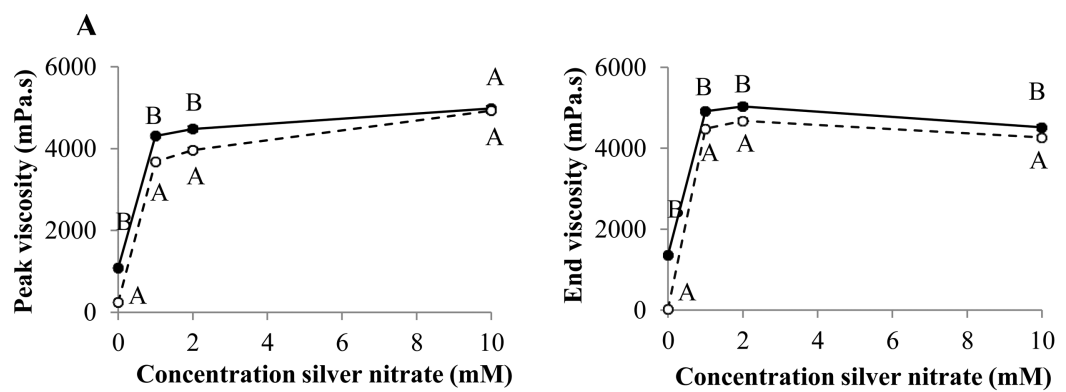

B
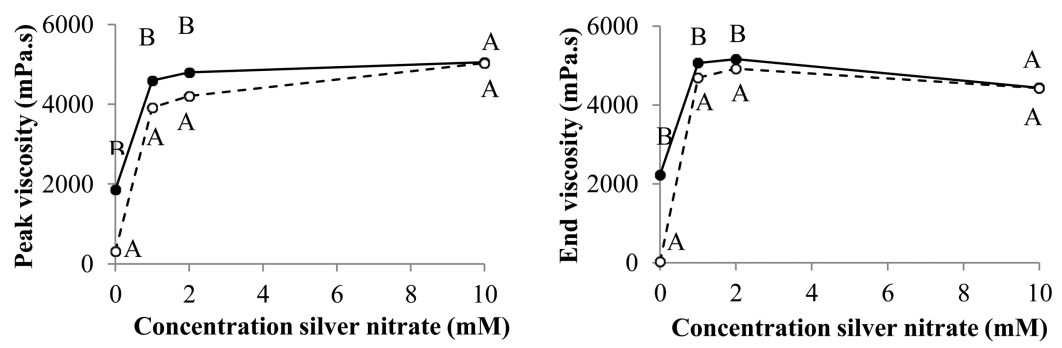

\section{C.}
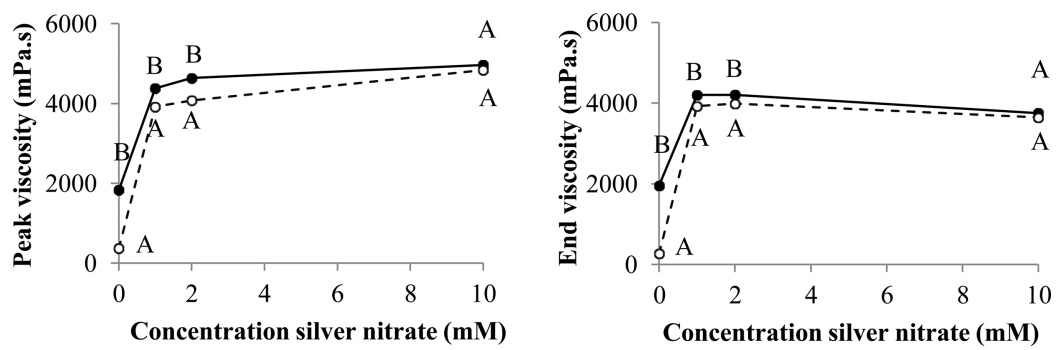

Figure 2. RVA peak (left) and end (right) viscosity readings of flour from mature (black spheres) and field sprouted (white spheres) wheat (12\% $\mathrm{dm}$ )-Sahara (A), Forum (B), and Tobak (C)-as a function of silver nitrate concentration. Viscosity values for the same silver nitrate concentration within the graph indicated by a different letter are significantly different $(p<0.05)$.

Table 3. Carbohydrate and Protein Characteristics of Flour from Mature and Field Sprouted Wheat of Sahara, Forum, and Tobak $^{a}$

\begin{tabular}{|c|c|c|c|c|c|c|}
\hline & Sahara $28 / 7$ & Sahara $20 / 8$ & Forum $28 / 7$ & Forum $25 / 8$ & Tobak $1 / 8$ & Tobak 2/9 \\
\hline total $\operatorname{starch}^{b}(\%)$ & $81.5 \pm 1.7 \mathrm{~A}$ & $81.7 \pm 1.6 \mathrm{~A}$ & $80.8 \pm 1.3 \mathrm{~A}$ & $82.3 \pm 1.0 \mathrm{~A}$ & $81.6 \pm 1.3 \mathrm{~A}$ & $82.0 \pm 1.3 \mathrm{~A}$ \\
\hline damaged $\operatorname{starch}^{b}(\%)$ & $7.8 \pm 0.2 \mathrm{~A}$ & $8.1 \pm 0.4 \mathrm{~A}$ & $6.9 \pm 0.2 \mathrm{~A}$ & $6.6 \pm 0.2 \mathrm{~A}$ & $7.0 \pm 0.4 \mathrm{~A}$ & $7.0 \pm 0.2 \mathrm{~A}$ \\
\hline glucose $^{b}(\mathrm{mg} / \mathrm{g})$ & $0.52 \pm 0.02 \mathrm{~A}$ & $0.42 \pm 0.01 \mathrm{~B}$ & $0.40 \pm 0.02 \mathrm{~A}$ & $0.33 \pm 0.02 \mathrm{~B}$ & $0.44 \pm 0.04 \mathrm{~A}$ & $0.38 \pm 0.01 \mathrm{~A}$ \\
\hline maltose $^{b}(\mathrm{mg} / \mathrm{g})$ & $0.66 \pm 0.07 \mathrm{~A}$ & $0.40 \pm 0.06 \mathrm{~B}$ & $0.54 \pm 0.04 \mathrm{~A}$ & $0.39 \pm 0.05 \mathrm{~B}$ & $0.56 \pm 0.11 \mathrm{~A}$ & $0.47 \pm 0.06 \mathrm{~A}$ \\
\hline maltotriose $^{b}(\mathrm{mg} / \mathrm{g})$ & $0.30 \pm 0.12 \mathrm{~A}$ & $0.23 \pm 0.07 \mathrm{~A}$ & $0.23 \pm 0.05 \mathrm{~A}$ & $0.25 \pm 0.07 \mathrm{~A}$ & $0.39 \pm 0.01 \mathrm{~A}$ & $0.21 \pm 0.09 \mathrm{~B}$ \\
\hline maltotetraose $^{b}(\mathrm{mg} / \mathrm{g})$ & $0.34 \pm 0.11 \mathrm{~A}$ & $0.30 \pm 0.06 \mathrm{~A}$ & $0.32 \pm 0.08 \mathrm{~A}$ & $0.36 \pm 0.11 \mathrm{~A}$ & $0.62 \pm 0.08 \mathrm{~A}$ & $0.32 \pm 0.12 \mathrm{~B}$ \\
\hline maltopentaose $^{b}(\mathrm{mg} / \mathrm{g})$ & $0.84 \pm 0.03 \mathrm{~A}$ & $0.75 \pm 0.03 \mathrm{~A}$ & $0.81 \pm 0.02 \mathrm{~A}$ & $0.77 \pm 0.03 \mathrm{~A}$ & $1.27 \pm 0.09 \mathrm{~A}$ & $1.00 \pm 0.08 \mathrm{~B}$ \\
\hline $\operatorname{protein}^{b}(\%)$ & $9.7 \pm 0.2 \mathrm{~A}$ & $10.5 \pm 0.1 \mathrm{~B}$ & $9.9 \pm 0.1 \mathrm{~A}$ & $10.5 \pm 0.1 \mathrm{~B}$ & $9.2 \pm 0.1 \mathrm{~A}$ & $9.5 \pm 0.1 \mathrm{~B}$ \\
\hline total $\mathrm{AX}^{b, c}(\%)$ & $2.3 \pm 0.1 \mathrm{~A}$ & $2.3 \pm 0.1 \mathrm{~A}$ & $2.3 \pm 0.1 \mathrm{~A}$ & $2.4 \pm 0.1 \mathrm{~A}$ & $2.2 \pm 0.1 \mathrm{~A}$ & $2.2 \pm 0.1 \mathrm{~A}$ \\
\hline WEAX $^{b, d}(\%)$ & $0.53 \pm 0.01 \mathrm{~A}$ & $0.72 \pm 0.02 \mathrm{~B}$ & $0.49 \pm 0.04 \mathrm{~A}$ & $0.65 \pm 0.02 \mathrm{~B}$ & $0.65 \pm 0.04 \mathrm{~A}$ & $0.89 \pm 0.02 \mathrm{~B}$ \\
\hline $\operatorname{avDP}^{e}$ WEAX & $150 \pm 1 \mathrm{~A}$ & $189 \pm 4 \mathrm{~B}$ & $153 \pm 11 \mathrm{~A}$ & $224 \pm 6 \mathrm{~B}$ & $184 \pm 11 \mathrm{~A}$ & $175 \pm 4 \mathrm{~A}$ \\
\hline
\end{tabular}

${ }^{a}$ Values are averages with standard deviations on triplicate measurements. Values of the same variety in the same row of the table followed by a different letter are significantly different $(p<0.05) .{ }^{b}$ Expressed on dry matter basis. ${ }^{c}$ AX: arabinoxylan. ${ }^{d}$ WEAX: water extractable arabinoxylan. ${ }^{e}$ avDP: average degree of polymerization.

${ }^{\circ} \mathrm{C}$, in the sprouted samples of Sahara, Forum and Tobak, respectively. This was concomitant with a drastic decline in peak viscosity and almost zero minimum and end viscosity values. Pasting temperatures of flour from sound and sprouted wheat were similar (approximately $59{ }^{\circ} \mathrm{C}$ ).

Next, silver nitrate was added to examine the rheological behavior of starch in flour from mature and sprouted wheat without the impact of $\alpha$-amylase during the analysis. As RVA measurements are more sensitive to low $\alpha$-amylase activity compared to $\mathrm{FN}^{20}$ different amounts of silver nitrate were used to set up a dose-response curve. In Figure 2 peak and end viscosity of the sound and sprouted wheat sample of each variety is given as a function of the concentration silver nitrate used during analysis. Addition of the lowest concentration of silver nitrate $(1.0 \mathrm{mM})$ already largely increased peak and end viscosity readings of flour from both mature and sprouted wheat. RVA readings still significantly differed between mature and sprouted samples. The difference between peak viscosity of 

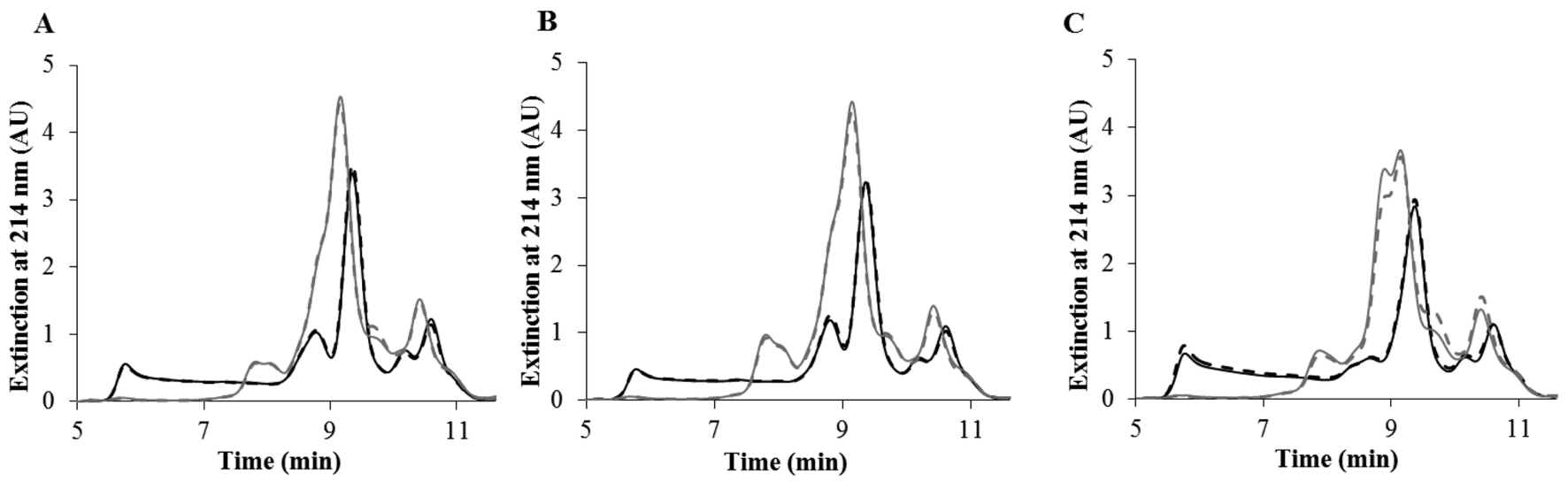

Figure 3. SE-HPLC chromatograms of flour proteins from mature (full lines) and field sprouted (dotted lines) wheat of Sahara (A), Forum (B), and Tobak (C) under nonreducing (black lines) and reducing (gray lines) conditions. AU are arbitrary units.

flour from mature and sprouted wheat decreased notably when higher concentrations of silver nitrate were added to more or less the same values, namely 4990 and $4930 \mathrm{mPa} . \mathrm{s}, 5055$ and $5030 \mathrm{mPa}$.s, and 4935 and $4875 \mathrm{mPa}$.s, for Sahara, Forum, and Tobak, respectively, which were for each variety not significantly different. A similar trend in decrease of the difference between flour from mature and sprouted wheat was observed for the end viscosity. After the addition of $10.0 \mathrm{mM}$ silver nitrate, the end viscosity values of flour from sprouted Forum and Tobak (4425 and $3680 \mathrm{mPa} . \mathrm{s}$, respectively) were not significantly different from the end viscosity measured in flour from mature wheat (4435 and $3720 \mathrm{mPa}$.s, respectively). However, a relatively small but significant difference was detected between end viscosity of flour from sprouted (4300 mPa.s) and mature Sahara (4480 mPa.s). To check whether the addition of silver nitrate was effective in reducing the $\alpha$-amylase activity, next to the RVA analyses, also $\alpha$-amylase activity measurements were performed. The remaining $\alpha$-amylase activity in flour samples of sprouted wheat after the addition of $10.0 \mathrm{mM}$ silver nitrate amounted to merely $0.5,0.3$, and $2.3 \%$ of the original $\alpha$-amylase activity $(93,113$, and $26 \mathrm{AU} / \mathrm{g}$ ) in flour of sprouted Sahara, Forum, and Tobak, respectively.

Analysis of Carbohydrate Content in Flour from Sound and Sprouted Wheat. Flour from the mature samples contained around $82 \%$ starch and the damaged starch content ranged from 6.9 to $7.8 \%$ (Table 3). Total and damaged starch content did not change significantly when PHS occurred. The total amount of measured malto-oligosaccharides in sound flour of the three varieties was in general very low (2.7, 2.3, and $3.3 \mathrm{mg} / \mathrm{g}$ flour for Sahara, Forum, and Tobak, respectively). Delaying harvest time of the samples resulted in a declining trend in the content of the different malto-oligosaccharides. Using gas chromatographic analysis, also a decrease in amount of free glucose was detectable (results not shown). The detected differences were small, but often significant.

Analysis of Protein Content, Extractability, and Molecular Weight Distribution of Proteins in Flour from Sound and Sprouted Wheat. The protein content of flour from grain at harvest maturity was $9.7,9.9$, and $9.2 \%$ for Sahara, Forum, and Tobak, respectively. When delaying harvest time, a slightly higher protein content in the flour samples was observed (Table 3).

To evaluate the extent of protein hydrolysis by peptidases, the amount of proteins of flour from mature and sprouted wheat extractable in SDS containing medium was measured. It was ascertained that there was no peptidase activity during the extraction procedure in the presence of $2 \%$ SDS. SDSEP readings of flour from mature Sahara, Forum, and Tobak were 84,78 , and $82 \%$, respectively. Delaying harvest time for Sahara and Forum did not cause any significant changes in SDS extractability of the proteins, while for Tobak a slightly higher SDSEP (86\%) was detected.

Next, qualitative changes in the molecular weight distribution of the proteins extracted in SDS containing medium in the presence and absence of reducing conditions were studied (Figure 3) to examine protein hydrolysis during field PHS. In general, extraction of proteins under nonreducing conditions did not reveal differences between flours from mature and sprouted wheat for any variety. After breaking disulfide bonds, also no alterations in the molecular weight distribution of the extracted proteins of flour from sprouted Sahara and Forum were detected. Proteins extracted from flour from sprouted Tobak wheat showed detectable differences in molecular weight distribution from mature flour, with an increase in lower molecular weight protein fragments at the expense of high molecular weight fragments.

Analysis of AX Content and Extractability in Flour from Sound and Sprouted Wheat. The total AX content in flour was similar for the three varieties and for the samples from mature and sprouted wheat $(2.2-2.3 \%$, Table 3$)$. On the contrary, the amount of WEAX-0.53, 0.49 , and $0.65 \%$ for mature wheat of Sahara, Forum, and Tobak, respectively-was slightly but significantly higher in flour from the sprouted wheat samples (0.72, 0.65 and $0.89 \%$, respectively), suggesting degradation of AX by endoxylanase activity during field PHS. It was ascertained that this degradation did not take place during the extraction procedure since endoxylanases were inactivated before the extraction by a heat treatment in ethanol. The avDP of WEAX was 1.3 and 1.5-fold higher in flour of field sprouted Sahara and Forum, respectively, whereas the avDP of WEAX measured in Tobak flour did not change significantly. The content of free arabinose measured with gas chromatographic analysis increased significantly from 0.04 to $0.12 \mathrm{mg} / \mathrm{g}$ in flour from mature and sprouted wheat of Tobak, respectively, whereas in flour of Sahara and Forum these concentrations remained constant $(0.03$ and $0.02 \mathrm{mg} / \mathrm{g}$, respectively). 


\section{DISCUSSION}

In this article, we studied the properties of starch, proteins, and AX of flour from sound and field sprouted wheat to determine whether the properties and hence inherent quality of these components is affected by PHS. We selected a sound (maximal FN) and a sprouted (low FN value) sample from three varieties (Sahara, Forum and Tobak) from a collection of samples obtained by growing these varieties under the same environmental conditions and by harvesting during the period from pre- to late maturity. The selected varieties showed a different susceptibility toward PHS: Sahara was more sensitive toward PHS as FN values decreased fast after harvest maturity concomitant with a strong increase in $\alpha$-amylase activity levels. On the contrary, Tobak was the most resistant of the tested varieties toward PHS as the drop in FN and rise in $\alpha$-amylase activity occurred slower and later when harvest time was delayed. Forum showed a medium susceptibility toward PHS during the tested period of sampling. ${ }^{16}$

Impact of PHS on Starch Functional Properties. In a first part, the functionality of starch from flour from sound and sprouted wheat was compared by examining its rheological behavior during Hagberg and RVA analysis. The parameters measured in these methods are strongly correlated to each other and to $\alpha$-amylase activity (results not shown), and are therefore often used to examine sprout damage in wheat. The mature samples were characterized by high FN values which classifies these flours in high quality grades for bread making purposes. $^{21}$ PHS was accompanied by a significant decline in FN to values at which wheat will be rejected for bread purposes at the time of sale $(<250 \mathrm{~s})$. Next, field sprouting caused a more or less similar decline (ca. $80 \%$ ) in peak viscosities for the three varieties, with a breakdown in viscosity to almost zero minimum viscosity readings. Pasting temperatures remained constant, but peak temperatures largely dropped during PHS, which was also noticed by Kulp et al. ${ }^{15}$ Thus, although the pasting process started at the same time in flour from mature and sprouted wheat, it appears that swelling of the starch granules in flour from sprouted wheat went faster and that the maximum swelling capacity was reached earlier due to the lower rigidity of the starch granules. In addition, end viscosity readings decreased to almost baseline values for flour from sprouted Sahara and Forum and to very small values for Tobak flour, indicating that the gelation capacity of flour was drastically decreased.

As FN and RVA viscosity readings are both dependent on the $\alpha$-amylase activity present in the samples, it is difficult to verify to which extent the observed changes in viscosity were caused by the (elevated) $\alpha$-amylase action during the analysis and to which extent these were caused by alterations in intrinsic starch properties which already took place during PHS in the field. Earlier attempts to investigate inherent starch pasting properties were performed by isolating the starch granules from sprouted wheat. However, remnants of $\alpha$-amylase activity associated with the granules could not be excluded. ${ }^{3,10}$ Therefore, in the current study, the $\alpha$-amylase action was blocked by adding silver nitrate which strongly inhibits $\alpha$ amylase during the test. ${ }^{20}$ Silver nitrate addition caused an increase in FN and RVA viscosity readings of flour from both mature and sprouted kernels. This indicates among others that even the low levels of $\alpha$-amylase in flour from sound wheat (5, 3 , and $3 \mathrm{AU} / \mathrm{g}$ in flour of Sahara, Forum, and Tobak, respectively), lead to noticeable starch breakdown under these conditions. After $\alpha$-amylase inactivation, flour from sprouted wheat was characterized by the same FN values as flour from mature wheat, suggesting that if changes in the inherent starch properties of field sprouted wheat had occurred, these changes were not large enough to make them differ significantly from those of sound wheat. Lorenz and colleagues ${ }^{10}$ also noticed elevated FN values after silver nitrate addition of flour from sound and laboratory sprouted wheat, nevertheless, substantial changes in starch properties had already occurred during laboratory sprouting of grain. In addition, in the present study, $\alpha$-amylase inactivation resulted in similar RVA profiles for flour from sound and sprouted wheat, indicating that the pasting and gelation properties of starch were not affected by PHS of wheat with a FN down to $150 \mathrm{~s}$. However, for RVA a higher silver nitrate concentration was needed than the one applied during the Hagberg test. The Hagberg method is clearly less sensitive to low levels of $\alpha$-amylase than the RVA due to fast heating in a short period of time. ${ }^{20}$ It is clear that addition of silver nitrate has a large impact on the $\alpha$-amylase activity, however, it is possible that this compound also affects other flour constituents contributing to a viscosity build-up. Investigating the effect of 2.0 and $10.0 \mathrm{mM}$ silver nitrate on starch and gluten revealed no alteration in their rheological properties measured in RVA (results not shown). Hence, the results described in the present study demonstrate that the changes observed in FN and RVA viscosity readings of flour from sprouted wheat were caused by starch degradation due to the action of elevated $\alpha$-amylase activities during the analysis and not by inherent changes in starch swelling, pasting, and gelation properties. In addition, the substantial increase in FN and RVA viscosity readings after the addition of silver nitrate strengthens the interest in a food grade inhibitor with the same potential as silver nitrate with the aim to upgrade the quality of flour from field sprouted wheat and to introduce this wheat in the food industry.

To confirm this finding, we checked whether small changes in starch content and structure had already occurred during severe PHS in the field, which were not detectable during the above-described viscometric analysis. Compared to flour from sound wheat, the total starch content in flour from the sprouted kernels was not lower which indicates that no starch was consumed yet by the germ for its growth during the germination process. Furthermore, similar damaged starch levels in flour from sound and sprouted wheat show that during PHS in the field no incipient hydrolysis of the starch molecules as a result of the action of amylolytic enzymes had already occurred. This is in line with the results from Kulp et al. ${ }^{15}$ who also did not detect inherent damage of starch granules during PHS in the field, but is in contrast with results from several other studies on laboratory sprouting. ${ }^{5,7,8}$ Also in contrast to these previous studies on laboratory sprouting, the amount of malto-oligosaccharides was not higher in flour from field sprouted wheat. Probably, the germination process in the present investigation had not progressed to the same extent. On the contrary, when comparing flour from mature and sprouted wheat, a small decrease in the content of different malto-oligosaccharides was detected. Possibly, this decrease illustrates the consumption of these saccharides stored in the scutellum for respiration and growth of the germ, ${ }^{1,22}$ as germination was already visible in some kernels by the elongation of the coleoptile and radicles. These sugars are presumably consumed primarily before the onset of enzymatically degradation of endosperm starch and transportation of degradation products toward the germ. However, it needs to be 
emphasized that the observed declines, although sometimes significant, were very small due to the low amounts initially present in flour from sound wheat. In conclusion, we can say that the structure and composition of starch granules in the sprouted wheat samples investigated here did not change during the PHS process that led to a flour FN of $150 \mathrm{~s}$.

The results on starch properties in this study are in contrast to what was found in previous work on laboratory sprouted grains. In these studies, extensive damage to starch granules was shown, causing altered water-binding, swelling, gelatinization, and gelation properties. ${ }^{7-10}$ Clearly, starch degradation takes place to a higher extent in laboratory sprouted grains than during PHS in the field due to the ideal conditions of germination applied in the laboratory. In the present study, degradation of starch due to $\alpha$-amylase activity had not progressed to the same stage as during laboratory sprouting and hence, starch in field sprouted wheat grain with a flour FN down to $150 \mathrm{~s}$ is still of a good quality.

Impact of PHS on Protein Characteristics. The quantity and quality of wheat gluten proteins determine the wheat quality and largely contribute to bread quality. ${ }^{22}$ So in this second part, it was investigated whether and to which extent changes in protein structure have already occurred during severe PHS in the field.

First, total protein content was examined in flour from sound and sprouted wheat. In general, this protein content was relatively low. Since the wheat yield in 2014 in Belgium was on average $10 \%$ higher than the quinquennial average, the available nitrogen in the soil has to be divided over a larger number of growing plants, leading to a lower protein content in the grain. ${ }^{23}$ Remarkably, for the sprouted samples, the protein content in the flour of the three varieties was higher, which is surprising as protein accumulation stops when the grain attains physiological maturity. ${ }^{24}$ A higher contamination of bran during milling could not explain these higher protein values, as no higher extraction yields or ash contents were detected in these samples. An increase in protein content has been reported repeatedly, however this increase is only relative, caused by the loss of carbohydrates due to respiration during germination, 25 which does not seem to be the case in the present study. On the contrary, other studies reported a slightly decreased protein content due to the consumption of low molecular weight nitrogen compounds necessary for the growth of the germ., ${ }^{2,12}$

In general, it is assumed that the gluten quality of flour from sprouted wheat is too low for optimal baking performance due to proteolytic hydrolysis of gluten proteins. ${ }^{2,11,12}$ For the Sahara and Forum varieties, no changes in the amount of proteins extractable in a SDS containing medium were observed during the period of sampling for field PHS, hence hydrolysis of flour proteins from sprouted wheat was not detectable. This observation was additionally confirmed in the SE-HPLC profiles of proteins extracted under nonreducing and reducing conditions, showing no changes in molecular weight distribution of flour proteins from sprouted wheat compared to sound wheat and, hence, no alteration in protein structure. These findings are in contrast to earlier studies, in which an increase in solubility and a shift in molecular weight distribution of flour proteins was noted after 2-3 days of germination., ${ }^{2,11,12}$ Presumably germination has progressed to a larger extent in these studies since it was performed under laboratory conditions. However, Lorenz et al., ${ }^{4}$ who studied flour proteins from field sprouted wheat, also mentioned incipient changes in gluten properties causing loss in extensibility. Nevertheless, these data should be interpreted with care since no measures were taken to inactivate peptidase activity during fractionation of gluten from flour, what presumably evoked this impaired quality.

In contrast to Sahara and Forum, the amount of flour proteins from sprouted Tobak extracted in SDS containing medium was slightly increased, indicating that peptidase attack caused incipient changes in protein structure. Nevertheless, this was not detectable in the SE-HPLC profile of proteins extractable under nonreducing conditions. The decrease in proteins of high molecular weight is presumably not observed due to the limited discriminating power of the analysis concerning the fractions eluting first. Hence, more information about possible proteolysis of Tobak proteins was obtained by reducing the disulfide bonds. A small shift from higher to lower molecular weight protein fragments extracted under reducing conditions was observed in flour from sprouted wheat, which was probably responsible for the increase in SDSEP of this flour. Unfortunately, for bread making, these high molecular weight proteins are essential for the strength of the gluten network and for optimal gas retention. ${ }^{22}$ Compared to the other two varieties, flour of the Tobak variety was characterized by the largest, although still small, increase in peptidase activity when delaying harvest time, which was presumably caused by a Fusarium contamination on the kernels. ${ }^{16}$ This microbial infection is probably responsible for the observed changes in protein structure rather than the sprouting process, as PHS in Forum and Sahara had progressed further and their proteins were still intact.

Impact of PHS on Arabinoxylan Characteristics. Similar to the total starch content, total AX levels in flour from sound and sprouted wheat of Sahara, Forum, and Tobak did not change significantly, indicating no consumption of degradation products of AX by the embryo yet. Nevertheless, incipient degradation of $\mathrm{AX}$ had started, observable by a significant increase in the amount of WEAX in flour. Previously, Dornez and colleagues ${ }^{19}$ also noticed that delaying harvest time in a harvest year with unfavorable weather conditions tended to increase the WEAX levels in wheat. Field sprouting causes elevated endogenous endoxylanase activity levels in flour, ${ }^{16}$ which could degrade aleurone and/or endosperm cell walls during PHS as these enzymes are not sensitive toward endogenous xylanase inhibitors. During germination, this degradation is necessary as cell walls form a physical barrier for $\alpha$-amylases and peptidases to access and degrade intracellular compounds for the developing embryo. ${ }^{1}$ Due to the high endogenous endoxylanase activity in flour of Tobak, ${ }^{16}$ flour from mature wheat was already characterized by higher WEAX levels in comparison to Sahara and Forum, and degradation of AX had even progressed further when harvest time was delayed. Also the amount of free arabinose was increased, suggesting the action of exoworking xylanolytic enzymes (arabinofuranosidases) in sprouted wheat of Tobak. That being said, one must be aware of possible increases in mycotoxins levels in flour from sprouted wheat, as the condition for the onset of PHS in the field are also advantageous for microbial growth.

The observed transformation of water unextractable AX (WUAX) into WEAX is generally considered as positive for bread making as WEAX is hypothesized to increase the viscosity of the dough aqueous phase, prolong the oven rise, and prevent early coalescence, in contrast to the destabilizing impact of WUAX. Nevertheless, further degradation of WEAX 
or solubilized AX fragments into smaller molecular weight fragments, on the contrary, diminishes viscosity again and, hence, leads to negative effects on bread loaves. ${ }^{26}$ For the flour samples of Sahara and Forum, the overall degradation of AX due to PHS resulted in a higher avDP of WEAX, suggesting that preferably WUAX molecules were hydrolyzed instead of WEAX. These changes would probably cause a positive effect on bread quality. The avDP of WEAX in flour from sprouted Tobak was comparable to that in flour from mature wheat, indicating that the solubilization of WUAX and further degradation of WEAX molecules occurred to a similar extent.

In conclusion, although the activity of certain enzymes was highly increased during PHS in the field, ${ }^{16}$ starch and proteins present in the grain did not yet undergo hydrolysis. This can be explained by the fact that the enzyme system needs a certain time to be fully operational. ${ }^{6}$ Second, mobilization of enzymes and subsequent breakdown of intracellular materials is hindered by the presence of cell walls. In the sprouted samples already incipient breakdown of $\mathrm{AX}$ was observed, which is considered not detrimental for the bread making process. Although PHS in the field is very heterogeneous and excessive degradation of storage compounds could have occurred in a marginal number of kernels, this was not detectable due to the large volume of wheat kernels milled into flour. Hence, the problems in the industry associated with flour from sprouted wheat with a flour $\mathrm{FN}$ down to $150 \mathrm{~s}$ are caused by the increased hydrolytic enzyme activity hydrolyzing flour constituents during dough processing and are not the result of enzyme induced inherent changes in the starch, gluten, and AX characteristics. Therefore, the present findings offer the opportunity to enhance the quality of flour from sprouted wheat by treatments reducing the enzymatic load. Furthermore, the results obtained in this study can strengthen the search for upgrading the economic value of sprouted grains with a flour FN down to $150 \mathrm{~s}$. As the structure and properties of starch and proteins of flour from preharvest sprouted wheat are still intact, these grains may serve as a good raw material source for the production of starch and gluten. However, further research is needed to clearly unravel the impact of field sprouted wheat during cereal-based processes.

\section{AUTHOR INFORMATION}

\section{Corresponding Author}

*Telephone: +32 163219 17; E-mail: christophe.courtin@ kuleuven.be.

\section{Funding}

Heleen Olaerts acknowledges Flanders' FOOD (Brussels, Belgium) for financial support within the framework of the REDUxAMYL project.

\section{Notes}

The authors declare no competing financial interest.

\section{ABBREVIATIONS USED}

avDP, average degree of polymerization; $\mathrm{AX}$, arabinoxylan; dm, dry matter; FN, falling number; PHS, preharvest sprouting; RVA, rapid visco analyzer; SDS, sodium dodecyl sulfate; SDSEP, amount of proteins extractable in sodium dodecyl sulfate; SE-HPLC, size exclusion high-performance liquid chromatography; WEAX, water extractable arabinoxylan; WUAX, water unextractable arabinoxylan

\section{REFERENCES}

(1) Meredith, P.; Pomeranz, Y., Sprouted grain. In Advances in Cereal Science and Technology, 7th ed.; Pomeranz, Y., Ed.; AACC: St. Paul, MN, 1985; pp $239-320$.

(2) Hwang, P.; Bushuk, W. Some changes in the endosperm proteins during sprouting of wheat. Cereal Chem. 1973, 50, 147-160.

(3) Ibrahim, Y.; D'Appolonia, B. Sprouting in hard red spring wheat. Bakers Digest 1979, 53 (5), 17-19.

(4) Lorenz, K.; Roewe-Smith, P.; Kulp, K.; Bates, L. Preharvest sprouting of winter wheat. II. Aminoacid composition and functionality of flour and flour fractions. Cereal Chem. 1983, 60 (5), $360-366$.

(5) Lukow, O. M.; Bushuk, W. Influence of germination on wheat quality. 1. Functional breadmaking and biochemical properties. Cereal Chem. 1984, 61 (4), 336-339.

(6) Kruger, J. E. Progress in the chemistry of some quality-affecting enzymes resulting from pre-harvest sprout damage. Cereal Research Communications 1980, 8 (1), 39-47.

(7) Dronzek, B. L.; Hwang, P.; Bushuk, W. Scanning electron microscopy of starch from sprouted wheat. Cereal Chem. 1972, 49, 232-239.

(8) Lineback, D.; Ponpipom, S. Effects of germination of wheat, oats, and pearl millet on $\alpha$-amylase activity and starch degradation. Starch Stärke 1977, 29 (2), 52-60.

(9) Simsek, S.; Ohm, J.-B.; Lu, H.; Rugg, M.; Berzonsky, W.; Alamri, M.; Mergoum, M. Effect of pre-harvest sprouting on physicochemical properties of starch in wheat. Foods 2014, 3 (2), 194-207.

(10) Lorenz, K.; Kulp, K.; Collins, F. Sprouting of cereal grains Effects on starch characteristics. Starch / Stärke 1981, 33 (6), 183187.

(11) Lukow, O. M.; Bushuk, W. Influence of germination on wheat quality. 2. Modification of endosperm protein. Cereal Chem. 1984, 61 (4), 340-344.

(12) Simsek, S.; Ohm, J.-B.; Lu, H.; Rugg, M.; Berzonsky, W.; Alamri, M. S.; Mergoum, M. Effect of pre-harvest sprouting on physicochemical changes of proteins in wheat. J. Sci. Food Agric. 2014, 94 (2), 205212.

(13) De Backer, E.; Gebruers, K.; Van den Ende, W.; Courtin, C. M.; Delcour, J. A. Post-translational processing of $\beta$-D-xylanases and changes in extractability of arabinoxylans during wheat germination. Plant Physiol. Biochem. 2010, 48 (2-3), 90-97.

(14) Meredith, P.; Jenkins, L. D. Communication to the editor. Amylase activity of sprout-damaged, malted and immature wheat. Cereal Chem. 1973, 50, 240-242.

(15) Kulp, K.; Roewe-Smith, P.; Lorenz, K. Preharvest sprouting of winter wheat. I. Rheological properties of flours and physicochemical characteristics of starches. Cereal Chem. 1983, 60 (5), 355-359.

(16) Olaerts, H.; Roye, C.; Derde, L. J.; Sinnaeve, G.; Meza, W. R.; Bodson, B.; Courtin, C. M. Evolution and distribution of hydrolytic enzyme activities during preharvest sprouting of wheat (Triticum aestivum) in the field. J. Agric. Food Chem. 2016, 64, 5644.

(17) AACC International Approved methods of analysis, 11th ed.; AACC International: St. Paul, MN. http://methods.aaccnet.org/toc. aspx (accessed May 9, 2016).

(18) AOAC, Official methods of analysis, 16th ed.; AOAC: Washington, DC, USA, 1995.

(19) Dornez, E.; Gebruers, K.; Joye, I. J.; De Ketelaere, B.; Lenartz, J.; Massaux, C.; Bodson, B.; Delcour, J. A.; Courtin, C. M. Effects of fungicide treatment, $\mathrm{N}$-fertilisation and harvest date on arabinoxylan, endoxylanase activity and endoxylanase inhibitor levels in wheat kernels. J. Cereal Sci. 2008, 47 (2), 190-200.

(20) Meredith, P. Effects of amylases and metals on the pasting properties of wheat flour, determined by amylograph and by Hagberg's Falling Number method. Cereal Chem. 1970, 47 (5), 483-491.

(21) Skerritt, J. H.; Heywood, R. H. A five-minute field test for onfarm detection of pre-harvest sprouting in wheat. Crop Sci. 2000, 40 (3), $742-756$.

(22) Delcour, J. A.; Hoseney, R. C. Principles of Cereal Science and Technology, third ed.; AACC: St. Paul, MN, 2010; p 270. 
(23) Atwell, W. An overview of wheat development, cultivation, and production. Cereal Foods World 2001, 46 (2), 59-62.

(24) Dupont, F. M.; Altenbach, S. B. Molecular and biochemical impacts of environmental factors on wheat grain development and protein synthesis. J. Cereal Sci. 2003, 38 (2), 133-146.

(25) Lorenz, K.; D'Appolonia, B. Cereal sprouts: composition, nutritive value, food applications. C R C Critical Reviews in Food Science and Nutrition 1980, 13 (4), 353-385.

(26) Courtin, C. M.; Delcour, J. A. Arabinoxylans and endoxylanases in wheat flour bread-making. J. Cereal Sci. 2002, 35 (3), 225-243. 